\title{
18ß-glycyrrhetinic Acid Protects against Staphylococcus aureus Infection by Regulating the NF-кB Pathway
}

\author{
Chunhui Rao', Zhuping Hong², Yuanyuan $\mathrm{Yao}^{3}$, Gang Zheng ${ }^{4}$, Saisai Wang ${ }^{3, *}$ \\ 'Department of Colorectal Surgery, Hangzhou Hospital of Traditional Chinese Medicine, Guangxing Hospital Affiliated to Zhejiang \\ University of Traditional Chinese Medicine, Hangzhou, P. R. CHINA. \\ ${ }^{2}$ Pharmaceutical Informatics Institute, College of Pharmaceutical Sciences, Zhejiang University, Hangzhou, P.R. CHINA. \\ ${ }^{3}$ Department of Colorectal Surgery, The First Affiliated Hospital, Zhejiang University School of Medicine, Hangzhou, P. R. CHINA. \\ ${ }^{4}$ Department of Cardiology, The Second Affiliated Hospital, Zhejiang University School of Medicine, Hangzhou, P. R. CHINA.
}

\begin{abstract}
Background: $18 \beta$-glycyrrhetinic acid $(18 \beta-\mathrm{GA})$ is reported to possess various pharmacological properties of which anti-inflammatory activities has been widely explored. However, the role of 18ß-GA in Staphylococcus aureus (SA) infection has not been investigated. The aim of the present study was to explore the effects of $18 \beta-G A$ on the SA infection especially the SA-induced Acute lung injury (ALI) and its related mechanisms. Material and methods: We infected the mice or cells with SA and then detected the survival rates of mice, bacterial burden and production of proinflammatory cytokines both in vitro and in vivo. We then detected the High-mobility group box 1 (HMGB1) expression by RT-qPCR and Western blotting. The effects on NF-kB activation was also determined by Western blotting and luciferase assay. Results: 18ß-GA could significantly improve the survival rate of SA-infected mice, reduce bacterial burden, suppress infiltration of inflammatory cells and reduce secretion of IL-1 $\beta$, IL- 6 and TNF- $\alpha$ both in lung tissues and cells. 18ß-GA treatment decreased high-mobility group box 1 (HMGB1) expression induced by SA infection and neutralizing of HMGB1 could improve the survival rate of mice induced by SA, implying that 18B-GA protected SA infection through down-regulating HMGB1 expression. Finally, we demonstrated that $18 \beta-$ GA inhibited the NF-kB activation. Conclusion: Taken together, our preliminary study suggested that $18 \beta-G A$ provided protective effects against $S A$ infection via its antiinflammatory properties possibly through down-regulating the HMGB1/NF-KB activation.
\end{abstract}

Key words: 18ß-glycyrrhetinic acid, Staphylococcus aureus, Acute lung injury, HMGB1, NF-kB.

\section{INTRODUCTION}

Staphylococcus aureus (SA) is a gram-positive bacterium that can cause a wide spectrum infection from mild infection of superficial skin to severe infection such as sepsis or septic shock. ${ }^{1}$ Lung is a common site of SA infection. Acute lung injury (ALI) is a complex syndrome characterized by hypoxemia, extensive pulmonary edema, tiny atelectasis, intrapulmonary hemorrhage and severely impaired gas exchange. ${ }^{2}$ The mechanisms involved in ALI including uncontrolled and excessive production of inflammatory mediators including cytokines, chemokines, adhesion molecules and bioactive lipid products. ${ }^{3}$ The primary cause of ALI is bacterial infection, in which SA is one of the most common pathogens. Despite the progress and improvements made in treatment, the mortality of pneumonia with ALI is still high. Strategies such as reducing bacterial load and attenuating exaggerated inflammation are under extensive evaluation. ${ }^{4}$
Submission Date: 12-11-2018; Revision Date: 09-12-2018; Accepted Date: 18-01-19.

DOI: 10.5530/ijper.53.2s.60 Correspondence: Dr. Saisai Wang, Department of Colorectal Surgery, The First Affiliated Hospital, Zhejiang University School of Medicine, 79 Qingchun Road, Hangzhou, Zhejiang 310003 , P.R. CHINA.

Phone: +86-057118767126425

E-mail: saisaiwang@zju. edu.cn

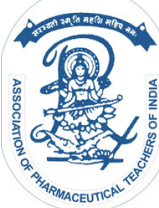

www.ijper.org 
$18 \beta$-glycyrrhetinic acid (18 $\beta$-GA), a major component of Glycyrrbiza glabra has been demonstrated to have several pharmacological effects including anti-inflammatory, anti-ulcer, anti-viral, antioxidant and hepatoprotective properties. ${ }^{5-8} 18 \beta-G A$ has been found to inhibit the LPS-induced inflammatory response in macrophages and increase the survival rate in LPS-induced ALI. ${ }^{9-10}$ Even in Methicillin-resistant Staphylococcus aureus (MRSA) infection, 18ß-GA could also attenuate the skin lesion and decrease the virulence gene expressions. ${ }^{11} 18 \beta-G A$ also exhibits the protective activities in inflammatory liver injury. ${ }^{12-14} 18 \beta-G A$ has been showed to attenuate the inflammation in SA-induced ALI. ${ }^{15}$ However, the anti-inflammatory effects and molecular mechanisms were unclear.

High-mobility group box 1 (HMGB1) is one of the members of Damage-associated molecular pattern (DAMP) and is associated with delayed and sustained release during infection. ${ }^{16}$ HMGB1 is highly released after SA infection and anti-HMGB1 could ameliorate the pulmonary damage, ${ }^{17}$ suggesting that HMGB1 might be a target in patients with pneumonia caused by SA.

The main objective of our study is to explore the effects of $18 \beta-G A$ on the SA infection, especially the induced ALI and the underlying mechanisms. Our study demonstrated that $18 \beta-G A$ provided protective effects against SA infection via its anti-inflammatory properties possibly through down-regulating the HMGB1/NF- $x \mathrm{~B}$ activation.

\section{MATERIALS AND METHODS}

\section{Chemicals and Mice}

$18 \beta$-glycyrrhetinic acid (18ß-GA) was obtained from Sigma-Aldrich (St. Louis, MO, USA) and dissolved in dimethyl sulfoxide (DMSO). Stock solutions were prepared at a concentration of $20 \mathrm{mg} / \mathrm{ml}$ and were diluted in medium to the appropriate concentrations indicated in each experiment. C57BL/6 mice (Shanghai Laboratory Animal Company, Shanghai, China) weighing between 16-18 g, age of 6-8 weeks were used. Mice were housed in a specific pathogen-free facility at Zhejiang University School of Medicine. Animals were treated in accordance with the Guide for the Care and Use of Laboratory Animals ( $8^{\text {th }}$ edition, National Academies Press). This study was approved by the Animal Experimental Ethical Inspection of the First Affiliated Hospital, College of Medicine, Zhejiang University (Permit number is 2017-475).

\section{Bacterial Strains and Growth Conditions}

The SA strain was a kind gift from professor FengXu, the Second Affiliated Hospital, Zhejiang University School of Medicine. ${ }^{1}$ The bacteria were allowed to grow at $37^{\circ} \mathrm{C}$ in Tryptone Soya Broth (TSB) medium. SA stocks were kept at $-80^{\circ} \mathrm{C}$ in TSB medium supplemented with $50 \%$ (vol/vol) of glycerol. Overnight cultures of SA were reinoculated into fresh TSB and grown to a log phase and then quantified according to a OD600-based bacterial growth curve and Colony forming units (CFU) assay. ${ }^{18}$

\section{Cell Culture and Treatment}

Human macrophage RAW264.7 cells and human liver hepatoma cells (HepG2) were obtained from American Type Culture Collection (ATCC, University Boulevard, Manassas, VA) and were cultured in DMEM supplemented with $10 \% \mathrm{FBS}, 100 \mathrm{U} / \mathrm{mL}$ penicilli and 100 $\mathrm{mg} / \mathrm{mL}$ streptomycin in a $5 \% \mathrm{CO}_{2}$ humidified incubator at $37^{\circ} \mathrm{C}$. All the cells were pretreated with $18 \beta-\mathrm{GA}$ or DMSO at a final concentration of $20 \mu \mathrm{M} 2 \mathrm{~h}$ before infected with SA at a Multiplicity of infection (MOI) of 10 and then continued culture for the indicated times.

\section{Mice Infection Model}

A SA-induced ALI mice model was established as previously reported. ${ }^{19-21}$ In brief, mice were randomly divided into three groups: group PBS: mice were intraperitoneally (i.p.) injected with DMSO $2 \mathrm{~h}$ prior to mock infection with PBS; group PBS+SA: mice were i.p. injected with DMSO $2 \mathrm{~h}$ prior to infection with $\mathrm{SA}\left(1 \times 10^{8}\right.$ CFU); group 18ß-GA + SA: mice were i.p. injected with $50 \mathrm{mg} / \mathrm{kg} 18 \beta-G A 2 \mathrm{~h}$ prior to SA infection. Mice were anesthesiaded by $1.5 \%$ pentobarbital $(5 \mu \mathrm{L} / \mathrm{g}$ per mouse) and then $40 \mu \mathrm{L}$ of SA or phosphate buffered saline (PBS) was inoculated directly intratracheally (i.t.) into the mice.

All the mice were used to perform survival rate, histological and molecular biological examination. The survival rate experiment was performed by examining them every 6 h up to 3 days.

To determine the role of HMGB1 in SA infection, mice were intraperitoneally injected with either $600 \mu \mathrm{g}$ antiHMGB1 neutralizing antibody (Santa Cruz Biotechnology, Santa Cruz, CA) or non-immune rabbit IgG in 500 $\mu \mathrm{L}$ PBS $1 \mathrm{~h}$ before SA infection.

\section{Histopathological Changes Examination}

Lungs and livers of the mice were fixed in $4 \%$ paraformaldehyde for $24 \mathrm{~h}$ at $4^{\circ} \mathrm{C}$, then embedded in paraffin. 4 $\mu \mathrm{M}$ sections were performed using a rotary microtome 
(Leica RM2016, Shanghai Leica Instruments, Shanghai, China) for Hematoxylin and Eosin (H\&E) staining. After staining, histopathological changes in the lung and liver tissues were observed under a light microscope.

\section{SA quantification in the Lung Homogenate (LH) and RAW264.7 Cells}

To determine the CFUs of the lung tissues after infection, whole lungs were extracted and homogenized in 1 $\mathrm{mL}$ PBS before $100 \mu \mathrm{L}$ of the homogenates were serially diluted 1:10 in PBS and plated on TSB agar to determine lung CFUs.

For CFUs of the RAW264.7 cells, cell culture medium was collected, and cells were lysed with $0.1 \%$ Triton X-100 to release live intracellular bacteria. The total samples were serially diluted 1:10 in PBS and plated on TSB plates to determine the number of CFUs.

\section{RNA Isolation and RT-PCR}

RNA was extracted from lung homogenates and RAW264.7 cells with the Kangwei RNA easy kit (CWBIO, China). After isolation, RNA samples were treated with DNase I for 60 min at room temperature to remove contaminating DNA. Real-time PCR was performed with ABI7500 and a SYBR Primer Script TM RT Reagent Kit (TaKaRa, Japan) following the manufacturer's instructions. The sequences for the primers were listed in Table 1. $\beta$-actin RNA was used as an internal control to normalize the data. Quantification was conducted via the comparative cycle threshold method.

\section{Cytokine Assays}

The production of proinflammatory cytokine in the Bronchial Alveolar Lavage Fluid (BALF) and RAW264.7 cells culture medium were detected by ELISA. BAL was performed as previously reported. Briefly, 800 microliters of PBS were instilled into the lung of the mice through the trachea and then carefully removed three times. BAL cells were collected and subjected to a blinded manual cell count. Supernatants of BAL were collected to measure the cytokine production. The concentrations of cytokines in the cell supernatants and BALF were measured using mice ELISA kits for IL-1 $\beta$, IL-6 and TNF- $\alpha$ (eBioscience, USA).

\section{ALT and AST Leakage}

The production of ALT and AST were determined with commercial kits purchased from the Institute of Biological Engineering of Nanjing Jiancheng (Nanjing, China) according to the manufacturer's instructions.

\section{Western Blotting}

Cells were rinsed with cold PBS and total cellular lysates were prepared using the RIPA buffer (CST, Danvers, MA) supplemented with phenyl methyl sulfonyl fluoride (PMSF, USA) and protease inhibitor cocktail. The protein concentrations were measured with the BCA Protein Assay Kit (CWBIO). The protein samples (20 $\mu \mathrm{g})$ were separated by $10 \%$ SDS-PAGE and transferred to PVDF membranes (Millipore, Billerica, MA, USA). The membranes were blocked with 5\% skimmed milk for $1 \mathrm{~h}$ at room temperature and probed with primary antibodies against phosphorylated or total proteins for p65, p38, JNK, ERK42/44 and HMGB1 (CST, Danvers, MA). After washing with TBST for three times, membranes were incubated with the HRP-conjugated secondary antibodies (Lianke, Hangzhou, China). ECL reagent were used to detect the immunoreactive bands on a digital image system (FluorChem E; Proteinsimple, Santa Clara, CA, USA).

\section{Luciferase Assay}

RAW264.7 cells were seeded in 96-well plate at a density of $1 \times 10^{4}$ per well 1 day prior to transfection. Cells were transient transfected with $\mathrm{NF}-\varkappa \mathrm{B}$ luciferase reporter plasmid with Lipofectamine 2000 (Invitrogen, Carlsbad, CA) following the manufacturer's instructions. After $24 \mathrm{~h}$ post transfection, cells were pretreated with $18 \beta$ GA or DMSO at a final concentration of $20 \mu \mathrm{M} 2 \mathrm{~h}$ before infected with SA. After $24 \mathrm{~h}$, cells were collected, and the luciferase activities were determined by using the Bright-Glo luciferase assay system (Promega Corp., Madison, WI).

\section{Statistical Analysis}

All data were analyzed using GraphPad Prism 5 (GraphPad InStatSoftware, San Diego, CA, USA). Results were presented as mean \pm SEM with at least three indepen-

\begin{tabular}{|c|c|c|}
\hline \multicolumn{3}{|c|}{ Table 1: Primer Sequences for Real-Time PCR. } \\
\hline Targets & Forward primer (5' $\mathbf{3}^{\prime}$ ) & Reverse Primer (5' $\mathbf{3}^{\prime}$ ) \\
\hline$\beta$-actin & AGAGGGAATCGTGCGTGAC & CAATAGTGATGACCTGGCCGT \\
\hline IL-1 $\beta$ & CCTCCTTGCCTCTGATGG & AGTGCTGCCTAATGTCCC \\
\hline IL-6 & TTCCAGAAACCGCTATGA & GGTTGTCACCAGCATCAG \\
\hline TNF- $\alpha$ & AATAACGCTGATTTGGTGA & ACC CGTAGGGCGATTACA \\
\hline HMGB1 & GCTGACAAGGCTCGTTATGAA & CCTTTGATTTTGGGGCGGTA \\
\hline
\end{tabular}




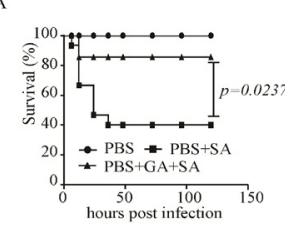

B
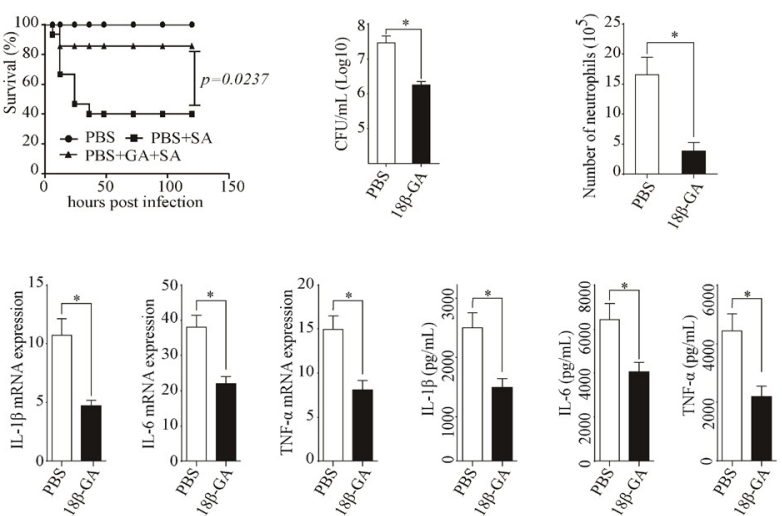

$\mathrm{D}$
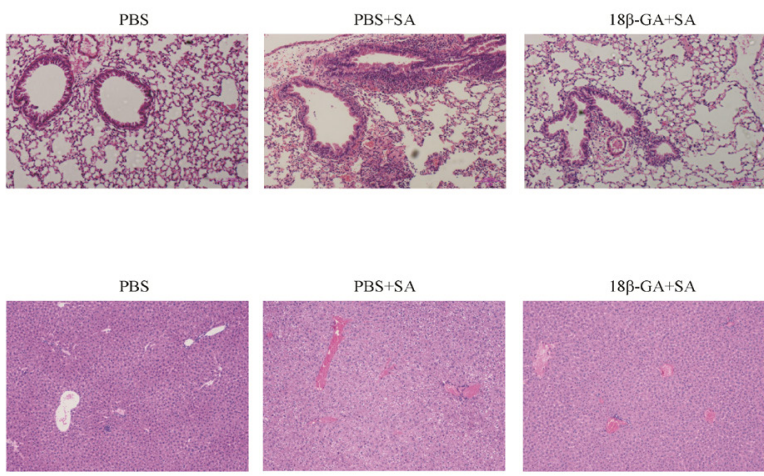

Figure 1: 18 $\beta$-GA protects mice against SA-induced ALI and the secondary liver injury. C57BL/6 mice were divided into three groups: PBS, PBS+SA, 18 $\beta-G A+S A$. Mice were intraperitoneal injected with either $18 \beta-\mathrm{GA}(50 \mathrm{mg} / \mathrm{kg})$ or PBS two $\mathrm{h}$ before infected with SA $\left(1 \times 10^{8} \mathrm{CFU}\right)$. Mice were kept alive for observation of their viability every $6 \mathrm{~h}$ for $120 \mathrm{~h}(\mathrm{n}=15$ per group) or sacrificed $12 \mathrm{~h}$ post infection (B-F, $n=4-8$ per group). (A) survival rate was determined (B) CFU counts in LH samples (C) mRNA expressions and protein production of IL-1 $\beta$, IL-6 and TNF-a in lung tissues and BAL fluid, respectively (D) HE staining of lung tissues $(\times 100)(E)$ numbers of neutrophils in BAL fluid (F) HE staining of liver tissues in each group ( $\times 100)$. The results represent the mean \pm SEM of three independent experiments. * $P<0.05$.

dent experiments. The Student $t$-test was used to estimate the significant differences between groups. Overall survival rates were calculated using the Kaplan-Meier method. The log-rank test was utilized to compare the survival rates between groups. $P$ values $<0.05$ are considered to indicate a statistically significant difference.

\section{RESULTS}

18ß-GA Protects Mice against SA-induced ALI and the Secondary Liver Injury

To evaluate the effect of $18 \beta-G A$ on SA infection, C57BL/ 6 mice were pre-treated with $18 \beta-G A 2 \mathrm{~h}$ before infected with SA. First, we checked the survival rate of the mice after SA infection. As shown in Figure
1A, 18ß-GA treatment significantly improved the survival rate compared with $P B S$-treated mice $(P=0.0237)$. We speculated that this might be resulted from the decreased bacterial load and attenuated inflammation. As shown in Figure 1B, lung tissues from mice treated with $18 \beta-G A$ showed lower bacterial counts at $12 \mathrm{~h}$ post $\mathrm{SA}$ infection $(P=0.043)$. Furthermore, $18 \beta-\mathrm{GA}$ attenuated the SA-induced increase of TNF- $\alpha$, IL- $1 \beta$ and IL-6 mRNA expression in lung and protein secretion in BALF (Figure 1C, $P<0.05$ ). With the challenge of SA, lung tissues were significantly damaged with various histopathologic changes including interstitial edema and hemorrhage, alveolar wall thickenin and notable infiltration of neutrophils in the lung parenchyma and alveolar spaces. While $18 \beta-G A$ treatment relieved the lung damage (Figure 1D) accompanied with the lower neutrophils infiltration (Figure 1E, $P=0.0074$ ). We also investigated the liver histopathology. As shown in Figure 1F, SA infected mice showed the infiltration with cells at the peripheral regions of the blood vessels and $18 \beta-G A$ reduced the cells infiltration.

\section{$18 \beta-G A$ Exhibits Antimicrobial activity and Inhibits Proinflammatory Cytokine Production in SA Infected RAW264.7 Cells}

To evaluate the effects of $18 \beta-G A$ on SA infection in vitro, RAW264.7 macrophages were implied for SA infection. First, cells were pre-treated with different concentrations of $18 \beta-G A$ before infected with SA for $6 \mathrm{~h}$ and then bacterial numbers were determined.18 $\beta$-GA significantly inhibited bacterial growth compared with control medium in a dose-dependent manner (Figure $2 \mathrm{~A})$. Consistent with the in vivo results, pretreatment of $18 \beta-G A$ significantly decreased the IL- $1 \beta$, IL- 6 and TNF- $\alpha$ expression and production (Figure $2 \mathrm{~B}, P<0.05$ ).

\section{8 $\beta$-GA Stabilizes HepG2 Liver Cells During SA Challenge}

We also detected the leakage of ALT and AST in culture medium from HepG2 cells exposed to SA infection with or without $18 \beta-G A$ treatment. As presented in Figure 2C, 18 $\beta$-GA significantly inhibited the leakage of ALT and AST ( $P=0.028$ and 0.018 , respectively) compared with the PBS-treated cells after SA challenge.

\section{HMGB1 Expression was Down-Regulated after 18ß-GA Treatment}

HMGB1 has been reported to increase sepsis and is positively correlated with the severity of sepsis in animals. ${ }^{22}$ Thus, we detected the HMGB1 expression both in lung tissues and in RAW264.7 cells. As shown in Figure $3 \mathrm{~A}-3 \mathrm{D}$, the mRNA and protein levels of HMGB1 in tissues and cells were remarkably increased after SA 
A

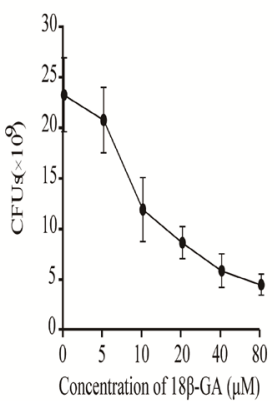

C
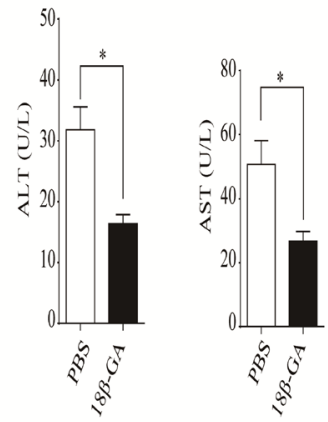

B

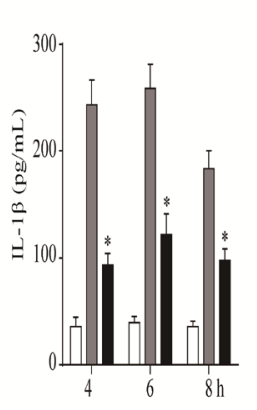

A

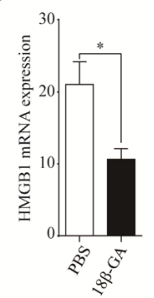

$\mathrm{C}$

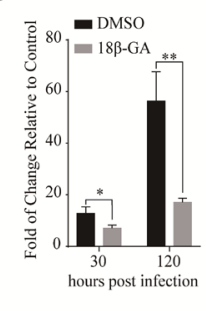

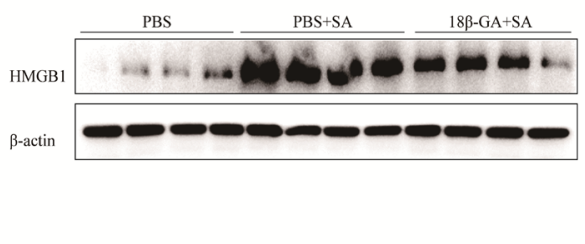

D

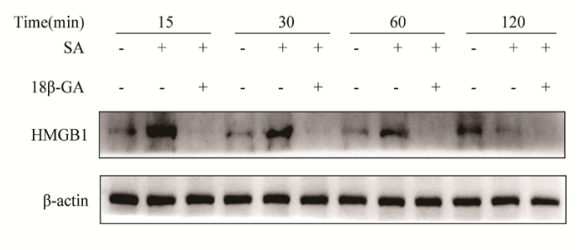

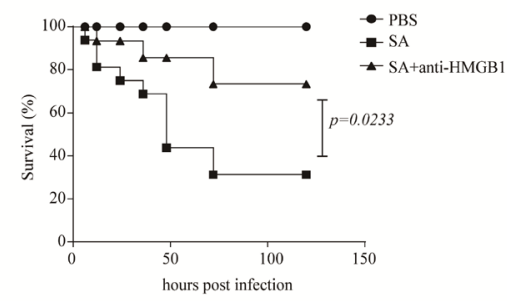

Figure 3: 18 $\beta-G A$ reduces HMGB1 expression and anti-HMGB1 increases the mice survival rates. Mice were intraperitoneal injected with either $18 \beta-G A(50 \mathrm{mg} / \mathrm{kg})$ or PBS $2 \mathrm{~h}$ before infected with SA (1×108 CFU). Mice were sacrificed $12 \mathrm{~h}$ post infection, (A) mRNA expression and (B) protein expression of HMGB1 in lung tissues were determined by RT-qPCR and Western blotting, respectively. RAW264.7 cells were pre-treated with $20 \mu \mathrm{M}$ of 18 $\beta$-GA $2 \mathrm{~h}$ before infected with SA. For the indicated hours post infection, cells were collected to determine the (C) mRNA expression and (D) protein expression of HMGB1. (E) Mice were intraperitoneally injected with either anti-HMGB1 neutralizing antibody or non-immune rabbit IgG before SA infection and the survival rate was determined. The results represent the mean \pm SEM of three independent experiments. ${ }^{* \star} P<0.01$, ${ }^{\star *} P<0.01$.

Therefore, to clarify the potential mechanism about its anti-inflammatory activity, the effects of $18 \beta-G A$ on the activation of $\mathrm{NF}-x \mathrm{~B}$ and MAPK signaling pathways were investigated. As shown in Figure 4A, SA infection significantly increased the phosphorylation of p65, p38, JN and ERK42/44 at 15, 30, 60 or $120 \mathrm{~min}$, as compared with control group. However, 18ß-GA treatment only markedly decreased the phosphorylation of p65 at 60 or $120 \mathrm{~min}$. We also assessed the NF- $x \mathrm{~B}$ activation using the luciferase reporter assay. After exposure to $18 \beta-G A, N F-x B$ activity was significantly decreased (Figure 4B, $P<0.0001$ ). These results suggested that alleviated effects of $18 \beta-G A$ on SA-induced inflammatory response were at least partially through regulation of $\mathrm{NF}-x \mathrm{~B}$ activation. 
A

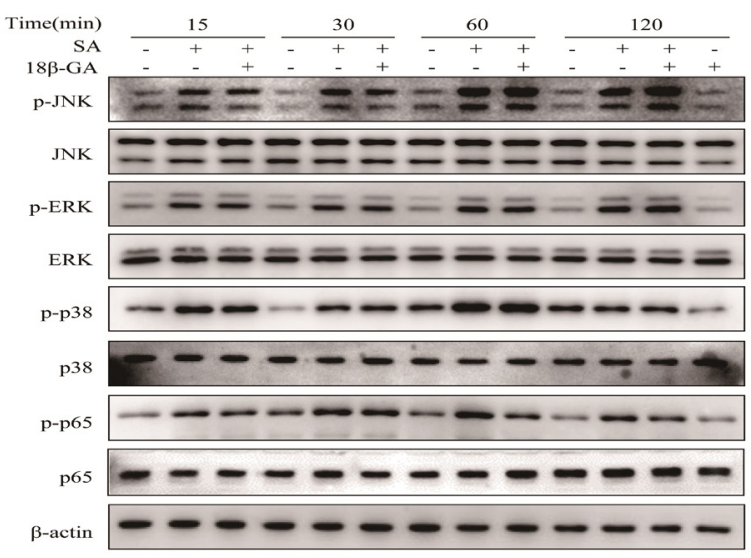

B

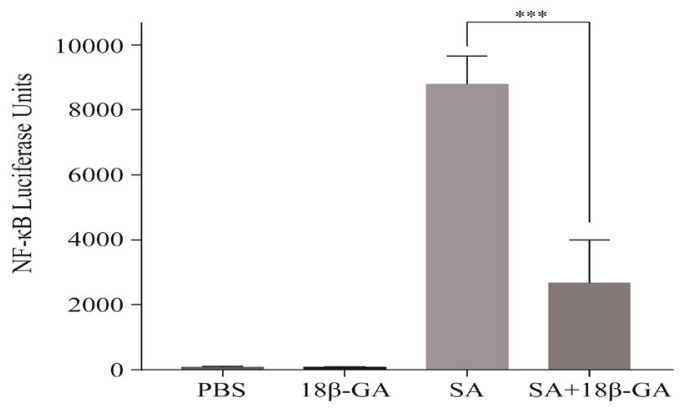

Figure 4: 18ß-GA inhibited the activation of NF-kB in RAW264.7 cells induced by SA. (A) RAW264.7 cells were pre-treated with $18 \beta-G A$ for $2 \mathrm{~h}$ before infected with SA. At the indicated times post infection, proteins were extracted to determine the p65, p-p65, p38, p-p38, JNK, p-JNK, ERK42/44, p-ERK42/44 and $\beta$-actin expression by Western blotting. (B) RAW264.7 cells were transfected with NF-kB luciferase reporter plasmid before cells were pre-treated with $18 \beta-G A$ or DMSO at a final concentration of $20 \mu \mathrm{M} 2 \mathrm{~h}$ before infected with SA. After 24 $h$, cells were collected and the luciferase activities were determined. The results represent the mean \pm SEM of three independent experiments. ${ }^{* \star *} P<0.001$.

\section{DISCUSSION}

The main findings of the present study were: (a) $18 \beta$ GA attenuated SA-induced acute lung injury and improved the survival rate of mice which might through reducing production of inflammatory mediators both in vivo and in vitro, (b) 18ß-GA decreased HMGB1 expression and neutralizing of HMGB1 can improve the survival rate of mice induced by SA, (c) 18 $\beta$-GA protected mice against secondary liver injury associated with SA-induced ALI and stabilized liver cells, (d) 18ß-GA down regulated NF- $x \mathrm{~B}$ inflammatory signaling pathway. These findings have potentially important implications for the treatment of SA infection or ALI.

$S$. aureus is a gram-positive bacterium which can cause a range of diseases including mild skin soft tissue injury, pneumonia, sepsis, endocarditi and deadly diseases such as toxic shock syndrome. ${ }^{23}$ Of these, pneumonia is one of the most prevalent diseases induced by SA infection with high mortality rates up to $8 \%$. SA infection induced $\mathrm{AL}$ and more severely, the Acute respiratory distress syndrome (ARDS), is a leading cause of morbidity and mortality in critically ill patients. Current treatments for ALI or ARDS are lacking, so it is important to explore the other possibilities.

$18 \beta$-GA has been reported to have several pharmacological effects including anti-tumor, anti-inflammation, anti-radiation and so on. Cao et al. reported 18ß-GA inhibits the initiation and progression of gastric tumors by ameliorating the inflammatory microenvironment through downregulation of COX-2 expression and inhibiting Wnt-1 expression. ${ }^{24}$ 18ß-GA significantly attenuates UV-induced skin photoaging mainly by virtue of its anti-oxidative and anti-inflammatory properties. ${ }^{25}$ The anti-inflammatory effects of $18 \beta-G A$ have been revealed in recent years. Studies have found $18 \beta-$ GA exhibits anti-inflammatory activities through inhibition of MIP- $1 \alpha$ production in acute propionibacterium acnes-induced inflammatory liver injury and inhibition of LPS/D-galactosamine-induced liver injury and prevention of free fatty acid-induced hepatic lipotoxicity. ${ }^{12-14} 18 \beta-G A$ also has been demonstrated to inhibit the LPS-induced inflammatory response in macrophages and increase the survival rate in LPS-induced ALI. ${ }^{9-10}$ In accordance with these studies, we revealed that 18ß-GA attenuated the inflammation in the SA induced ALI model.

Studies report that HMGB1 is highly expressed in many kinds of inflammatory disorders and contributes to the inflammation process. One study reported that HMGB1 expression is increased in the patients with conjunctivitis or blephariti and $18 \beta$-GA impairs antibody recognition of HMGB1 by direct binding to the protein thus pre-

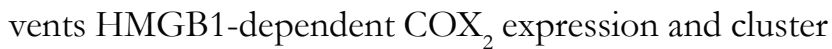
formation. ${ }^{26}$ In our study, we also found that $18 \beta-G A$ decreased the HMGB1 expression induced by SA infection, while the exact mechanisms was not detected further and which was the focus of our future study.

Clinical and experimental studies have shown that ALI is an early and fatal complication of septic shock ${ }^{27}$ and the TLR2/4-mediated NF- $x$ B activation could be an early molecular event leading to ALI during septic shock. ${ }^{28-29}$ $\mathrm{NF}-x \mathrm{~B}$ is a critical transcription factor in TLR-mediated signaling pathways ${ }^{30}$ and plays a critical role in regulation of expressions of a number of genes, including inflammatory cytokines such as HMGB1,TNF- $\alpha$ and IL- $1 \beta .{ }^{31-32} 18 \beta-G A$ has been found to attenuate NF- $x$ B activation to alleviate the inflammation. ${ }^{26}$ In our study, 
we found the same phenomenon that $18 \beta$-GA inhibited the activation of $\mathrm{NF}-x \mathrm{~B}$.

\section{CONCLUSION}

Taken together, we demonstrated that $18 \beta-G A$ protects mice against lung and liver injury after SA infection. The possible mechanisms are related to the decreased pro-inflammatory factors and HMGB1 expressions which might through the inhibition of NF- $x \mathrm{~B}$-regulated inflammation signaling pathways. Thus, $18 \beta-G A$ may have the potential to serve as a useful therapeutic agent for treating ALI caused by bacterial infection.

\section{ACKNOWLEDGEMENT}

The present study was supported by a grant from the Key Project of Natural Science Foundation of Zhejiang Province (LZ16H160003).

\section{CONFLICT OF INTEREST}

The authors declare no conflict of interest.

\section{ABBREVIATIONS}

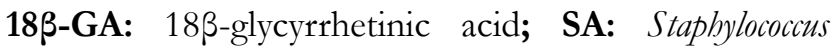
aureus; ALI: Acute lung injury; HMGB1: High-mobility group box 1 .

\section{REFERENCES}

1. Feng X, Maze M, Koch LG, Britton SL, Hellman J. Exaggerated Acute Lung Injury and Impaired Antibacterial Defenses During Staphylococcus aureus Infection in Rats with the Metabolic Syndrome. PLoS One. 2015;10(5):e0126906.

2. Zhang Y, Li X, Grailer JJ, Wang N, Wang M, Yao J, et al. Melatonin alleviates acute lung injury through inhibiting the NLRP3 inflammasome. J Pineal Res. 2016.

3. Fu J, Wang Y, Zhang J, Wu W, Chen X, Yang Y. Anti-inflammatory and antiapoptotic effects of oxysophoridine on lipopolysaccharide-induced acute lung injury in mice. Am J Transl Res. 2015;7(12):2672-82.

4. Xu F, Hu Y, Zhou J, Wang $X$. Mesenchymal stem cells in acute lung injury: are they ready for translational medicine?. J Cell Mol Med. 2013;17(8):927-35.

5. Mahmoud AM, Al Dera HS. 18beta-Glycyrrhetinic acid exerts protective effects against cyclophosphamide-induced hepatotoxicity: potential role of PPARgamma and Nrf2 upregulation. Genes Nutr. 2015;10(6):41.

6. Ishida T, Miki I, Tanahashi T, Yagi S, Kondo Y, Inoue J, et al. Effect of 18beta-glycyrrhetinic acid and hydroxypropyl gammacyclodextrin complex on indomethacin-induced small intestinal injury in mice. Eur J Pharmacol. 2013;714(1-3):125-31.

7. Maitraie D, Hung CF, Tu HY, Liou YT, Wei BL, Yang SC, et al. Synthesis, antiinflammator and antioxidant activities of 18beta-glycyrrhetinic acid derivatives as chemical mediators and xanthine oxidase inhibitors. Bioorg Med Chem. 2009;17(7):2785-92.

8. Hardy ME, Hendricks JM, Paulson JM, Faunce NR. 18beta-glycyrrhetinic acid inhibits rotavirus replication in culture. Virol J. 2012;9:96.

9. Wang CY, Kao TC, Lo WH, Yen GC. Glycyrrhizic acid and 18betaglycyrrhetinic acid modulate lipopolysaccharide-induced inflammatory response by suppression of NF-kappaB through PI3K p110delta and p110gamma inhibitions. J Agric Food Chem. 2011;59(14):7726-33.
10. Kim JK, Oh SM, Kwon HS, Oh YS, Lim SS, Shin HK. Anti-inflammatory effect of roasted licorice extracts on lipopolysaccharide-induced inflammatory responses in murine macrophages. Biochem Biophys Res Commun. 2006;345(3):1215-23.

11. Long DR, Mead J, Hendricks JM, Hardy ME, Voyich JM. 18beta-Glycyrrhetinic acid inhibits methicillin-resistant Staphylococcus aureus survival and attenuates virulence gene expression. Antimicrob Agents Chemother. 2013;57(1):241-7.

12. Xiao $\mathrm{Y}, \mathrm{Xu} \mathrm{J}$, Mao C, Jin M, Wu Q, Zou J, et al. 18Beta-glycyrrhetinic acid ameliorates acute Propionibacterium acnes-induced liver injury through inhibition of macrophage inflammatory protein-1alpha. J Biol Chem. 2010;285(2):1128-37.

13. Yoshida $T$, Abe K, Ikeda T, Matsushita T, Wake K, Sato T, et al. Inhibitory effect of glycyrrhizin on lipopolysaccharide and d-galactosamine-induced mouse liver injury. Eur J Pharmacol. 2007;576(1-3):136-42.

14. Wu X, Zhang L, Gurley E, Studer E, Shang J, Wang T, et al. Prevention of free fatty acid-induced hepatic lipotoxicity by 18beta-glycyrrhetinic acid through lysosomal and mitochondrial pathways. Hepatology. 2008;47(6):1905-15.

15. Li HE, Qu JZ, Yang ZQ, Dong J, Wang JF, Luo MJ, et al. Glycyrrhetinic acid protects mice from Staphylococcus aureus Pneumonia. Fitoterapia. 2012;83(1):241-8.

16. Sims GP, Rowe DC, Rietdijk ST, Herbst R, Coyle AJ. HMGB1 and RAGE in inflammation and cancer. Annu Rev Immunol. 2010;28:367-88.

17. Achouiti A, van der Meer AJ, Florquin S, Yang $\mathrm{H}$, Tracey KJ, van 't Veer $\mathrm{C}$, et al. High-mobility group box 1 and the receptor for advanced glycation end products contribute to lung injury during Staphylococcus aureus pneumonia. Crit Care. 2013;17(6):R296.

18. Oyama K, Kawada-Matsuo M, Oogai Y, Hayashi T, Nakamura N, Komatsuzawa H. Antibacterial Effects of Glycyrrhetinic Acid and Its Derivatives on Staphylococcus aureus. PLoS One. 2016;11(11):e0165831.

19. Qian J, Hu Y, Zhao L, Xia J, Li C, Shi L, et al. Protective role of adiposederived stem cells on Staphylococcus aureus-induced lung injury is mediated by Reglllgamma secretion. Stem Cells. 2016.

20. Xu F, Diao R, Liu J, Kang Y, Wang X, Shi L. Curcumin attenuates Staphylococcus aureus-induced acute lung injury. Clin Respir J. 2015;9(1):8797.

21. Xu F, Kang Y, Zhang H, Piao Z, Yin H, Diao R, et al. Akt1-mediated regulation of macrophage polarization in a murine model of Staphylococcus aureus pulmonary infection. J Infect Dis. 2013;208(3):528-38.

22. Huston JM, Wang H, Ochani M, Ochani K, Rosas-Ballina M, GallowitschPuerta M, et al. Splenectomy protects against sepsis lethality and reduces serum HMGB1 levels. J Immunol. 2008;181(5):3535-9.

23. Grundmann H, Aires-de-Sousa M, Boyce J, Tiemersma E. Emergence and resurgence of meticillin-resistant Staphylococcus aureus as a public-health threat. Lancet. 2006;368(9538):874-85.

24. Cao D, Jia Z, You L, Wu Y, Hou Z, Suo Y, et al. 18beta-glycyrrhetinic acid suppresses gastric cancer by activation of miR-149-3p-Wnt-1 signaling. Oncotarget. 2016;7(44):71960-73.

25. Kong SZ, Chen HM, Yu XT, Zhang X, Feng XX, Kang XH, et al. The protective effect of 18beta-Glycyrrhetinic acid against UV irradiation induced photoaging in mice. Exp Gerontol. 2015;61:147-55.

26. Cavone L, Muzzi M, Mencucci R, Sparatore B, Pedrazzi M, Moroni F, et al. 18beta-glycyrrhetic acid inhibits immune activation triggered by HMGB1, a pro-inflammatory protein found in the tear fluid during conjunctivitis and blepharitis. Ocul Immunol Inflamm. 2011;19(3):180-5.

27. Xu YZ, Zhang RY, Li CL, Yin X, Lv CJ, Wang YQ, et al. Dexmedetomidine attenuates acute lung injury induced by lipopolysaccharide in mouse through inhibition of MAPK pathway. Fundamental and Clinical Pharmacology. 2015;29(5):462-71.

28. Feng G, Sun B, Li TZ. Daidzein attenuates lipopolysaccharide-induced acute lung injury via toll-like receptor 4/NF-kappaB pathway. International Immunopharmacology. 2015;26(2):392-400.

29. Tianzhu Z, Shumin W. Esculin Inhibits the Inflammation of LPS-Induced Acute Lung Injury in Mice Via Regulation of TLR/NF-kappaB Pathways. Inflammation. 2015;38(4):1529-36.

30. Kawasaki T, Kawai T. Toll-like receptor signaling pathways. Front Immunol. 2014;5:461. 
Yang Q, Liu X, Yao Z, Mao S, Wei Q, Chang Y. Penehyclidine hydrochloride inhibits the release of high-mobility group box 1 in lipopolysaccharideactivated RAW264.7 cells and cecal ligation and puncture-induced septic mice. J Surg Res. 2014;186(1):310-7.
Chen N, Liu D, Soromou LW, Sun J, Zhong W, Guo W, et al. Paeonol suppresses lipopolysaccharide-induced inflammatory cytokines in macrophage cells and protects mice from lethal endotoxin shock. Fundam Clin Pharmacol. 2014;28(3):268-76

\section{PICTORIAL ABSTRACT}

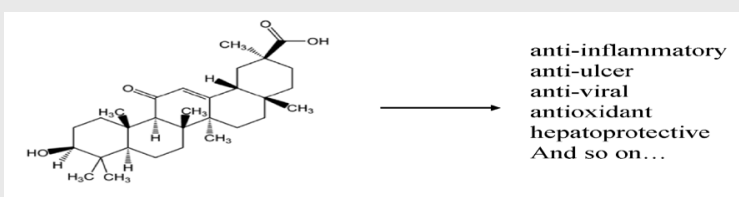

$18 \beta-$ glycyrrhetinic acid $(18 \beta-G A)$

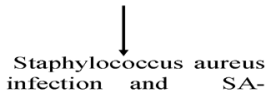

infection and $\mathrm{SA}$

induced

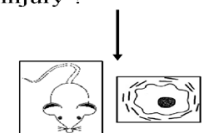

SA infection

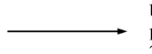

1.survival rates, bacterial proinflammatory cytokines 2. HMGB 1 expression

\section{ABOUT AUTHORS}

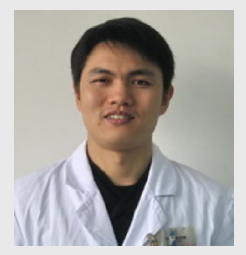
University School of Medicine.

\section{SUMMARY}

- $18 \beta$-glycyrrhetinic acid (18ß-GA) is reported to possess various pharmacological properties. In this study, we explored the effects of $18 \beta-\mathrm{GA}$ on the SA infection especially the SA-induced acute lung injury and its related mechanisms. Mice or cells were infected with SA both in vitro and in vivo and the survival rates of mice, bacterial burden and production of proinflammatory cytokines were detected. The expression of high-mobility group box 1 (HMGB1) and NF- $x \mathrm{~B}$ activation was further determined. We found that $18 \beta-\mathrm{GA}$ provided protective effects against $\mathrm{SA}$ infection via its anti-inflammatory properties possibly through down-regulating the HMGB1/NF- $x \mathrm{~B}$ activation, indicating that $18 \beta-G A$ may have the potential to serve as a useful therapeutic agent for treating ALI caused by bacterial infection.

Mr. Chunhui Rao, is a surgeon from Department of Colorectal Surgery, Hangzhou Hospital of Traditional Chinese Medicine, Hangzhou, China. He completed his Bachelor's degree in Gannan Medical University in 2004, currently he is pursuing his master degree in Zhejiang

Cite this article: Rao C, Hong Z, Yao Y, Zheng G, Wang S. 18 $\beta$-glycyrrhetinic Acid Protects against Staphylococcus aureus Infection by Regulating the NF-kB Pathway. Indian $\mathrm{J}$ of Pharmaceutical Education and Research. $2019 ; 53(2 S): s 151-s 158$ 